\title{
Lightweight Compliant Arm with Compliant Finger for Aerial Manipulation and Inspection
}

\author{
Alejandro Suarez, Guillermo Heredia and Anibal Ollero
}

\begin{abstract}
This paper presents the design and experimental validation of a compliant and lightweight 3-DOF robotic arm shoulder yaw, shoulder pitch and elbow pitch joints - equipped with a compliant finger module intended for aerial inspection and manipulation in contact with the environment. A simple transmission mechanism consisting in a pair of compression springs and a flange bearing is integrated in the shoulder pitch and elbow pitch joints between the servo shaft and the output frame. Joint deflection measurement with potentiometer allows joint torque but also contact force estimation and control. The low stiffness of the compliant finger has been exploited for soft collision detection and obstacle localization, in such a way that the contact forces do not significantly disturb the UAV. Fixedbase experiments have been performed with the arm, including the characterization of the compliant joints and the control of the contact force at wrist point.
\end{abstract}

\section{INTRODUCTION}

The current development in the UAV (Unmanned Aerial Vehicle) technology with autonomous helicopters and multirotors motivates the research in new areas and applications, where the ability of these platforms for reaching zones out of the range of ground robots is exploited. The field of the aerial manipulation considers the integration of robotic arms in an aerial platform [1][2] for the execution of tasks like assembly and structure construction [3], grasping and transportation [4], pick-and-place [5] or inspection [6] and maintenance of industrial facilities, reducing significantly the time, cost and resources required in the deployment of aerial robots with respect to other solutions.

Besides the problem of modeling and controlling an aerial manipulation system [7][8][9], it results necessary to design specific robotic arms suitable for this kind of vehicles. Low weight and inertia are the most immediate requirements due to the severe payload limitations and the convenience of reducing as much as possible the influence of arm's motion over UAV stability due to dynamic coupling. With this idea, the 5-DOF robotic arm proposed in [10] uses timing belts as transmission mechanism, placing the elbow and wrist servos at the base of the arm. The human-size arm described in [11] employs a linear actuator for the elbow joint, so the mass of the actuator is distributed along the upper arm link. A 2-DOF robotic arm built from Dynamixel servos is developed in [12] for object grasping and transportation with a quadrotor. Two 2-DOF robotic arms also built with Dynamixel servos are considered in [13] for on-flight valve turning with quadrotor.

Compliance is a highly desirable feature for robotic arms motivated by safety and efficiency reasons [14][15]. In the

A. Suarez (asuarezfm@us.es), G. Heredia (guiller@us.es) and A. Ollero (aollero@us.es) are with the Robotics, Vision and Control Group at the University of Seville (Spain). case of aerial manipulation, compliance prevents that contact forces are rigidly propagated from the contact point to the base of the UAV [16], enhances the protection of the actuators against shocks and impacts, and increases the reaction time of the platform for compensating oscillations in the positioning when there are motion constraints associated to contact or grasping situations. Compliance in robotic arms has been also exploited for collision detection [17] or payload estimation [11]. Some mechanisms for aerial grasping [18] and manipulation [19] with helicopter and multirotor UAVs have been developed. Dynamic modeling and control of manipulator arms with compliant joints has been studied in [20][21], although achieving accurate, fast position control for grasping on flight becomes a hard task, as accelerations in the UAV will cause deflections in the flexible joints.

The main contribution of this paper is the development and experimental validation of a low weight robotic arm with compliant joints and compliant finger intended for aerial manipulation and inspection applications with small-medium scale multi-rotors. The arm has been specifically designed for those tasks involving contact forces between the robotic arm and the environment such like peg-in-hole, sensor collocation or on flight grasping. The arm provides $3 \mathrm{DOFs}$ with torque estimation based on joint deflection measurement. In order to control the contact force, the torque-deflection characteristic is derived along with the force-torque relationships. A simple control scheme which considers arm kinematics, dynamics and the tasks it is intended to perform is described and tested experimentally in a fixed base test bench.

The rest of the paper is organized as follows. Section II introduces some considerations affecting the design of robot manipulators for aerial manipulation, presenting then the proposed design of the compliant arm-finger system. Section III deals with the torque-force estimation based on joint deflection measurement. Section IV covers the kinematics, dynamics and control of the arm. The experimental setup and results with the arm-finger system are presented in Section $\mathrm{V}$, while Section VI contains the conclusions of this work.

\section{COMPLIANT ARM AND FINGER SYSTEM}

\section{A. Design Considerations}

The design and development of robot manipulators for aerial manipulation applications imposes severe constraints that fixed base manipulators commercially available do not properly satisfy. First of all, the arm must be low weight and inertia in order to reduce as much as possible the influence of arms motion over the stability of the aerial vehicle due to dynamic coupling. This demands the use of materials with low mass density and high mechanical resistance for the frame such like aluminum or carbon fiber. As general criteria, the weight of the robotic arm must be around four times 
lower than the weight of the aerial platform (including the batteries), while its length when fully stretched should not exceed the perimeter of the propellers, so the mass unbalance does not cause high overloads in some of the motors. Also it is desirable that the ratio between the maximum lift load and the weight of the arm is close to one or higher. Mechanical robustness is a required feature for the frame parts in order to prevent unnecessary repairs or replacements in case the UAV crashes and the arm is affected by strong shocks or impacts.

Smart servos like Herkulex or Dynamixel are a suitable choice for the actuators, as they include the motor, gearbox, electronics, control and communications in a compact, high torque to weight ratio device, simplifying the mechanical design of the arm. The limitations in terms of estimation and control (update rates under $100 \mathrm{~Hz}$ ) and the lack of direct torque measurement of these devices make necessary the integration of some kind of compliant element between the servo shaft and the output link with a double functionality: transmission mechanism and torque estimation based on joint deflection measurement. Compliance provided by elastic elements like springs or elastomers increases safety in the aerial platform in situations involving physical interactions with the environment, but also the lifespan of the actuators, protecting them from impacts and overloads. Compression springs were preferred in this work rather than elastomers as this kind of materials may suffer from hysteresis or long term deformations. The ability of springs to absorb rapidly the energy of shocks and vibrations increases the bandwidth and therefore the mechanical tolerance of the joints, although at expenses of reducing the accuracy in the control. In this sense, it would be interesting having a damping element in parallel with the springs.

\section{B. Arm Design}

A picture of the developed 3-DOF robotic arm has been represented in Figure 1. The kinematic configuration consists in the shoulder yaw joint at the base followed by the shoulder pitch and elbow pitch joints. Note that this configuration simplifies the resolution of the inverse kinematics, and thus the control, providing a wide 3D workspace. The actuators are three Herkulex DRS-0101 servos from Dongbu Robot. The frame of the arm consists in sixteen parts manufactured by hand in anodized aluminum from flat profiles $(20 \times 2$ and $25 \times 2 \mathrm{~mm}$ ), and $8 \mathrm{~mm}$ hollow circular profile. Aluminum is a well suited material due to its low cost, low mass density (2.8 $\mathrm{g} / \mathrm{cm}^{3}$ ), and high mechanical resistance.
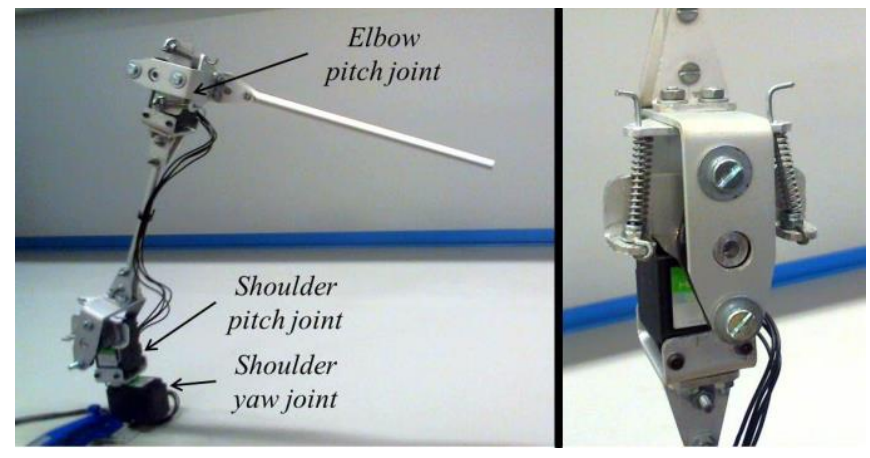

Figure 1. 3-DOF robotic arm (left) and compliant joint (right) integrated in shoulder pitch and elbow pitch joints.
The specifications of the arm, including the weight, size, payload, or rotation range for each joint, can be found on Table 1. The volume of operation corresponds to a hollow semi-sphere generated by the revolution around the shoulder yaw axis of the circular ring that represents the points reachable by the extreme of the forearm link. This allows the manipulator to reach a wide range of points under the base of the multirotor platform. The lengths of the upper arm and forearm links have been chosen in such a way that the wrist point is out of the range of the propeller in a medium-scale quadrotor when the arm is fully extended.

Table 1. Specifications of the 3-DOF compliant robot arm.

\begin{tabular}{|c|c|}
\hline Weight & $0.3[\mathrm{Kg}]$ \\
\hline Max. lift load & $0.2[\mathrm{Kg}]$ \\
\hline Size & $\begin{array}{c}\text { Upper arm: } \mathrm{L}_{1}=0.2[\mathrm{~m}] \\
\text { Forearm: } \mathrm{L}_{2}=0.2[\mathrm{~m}]\end{array}$ \\
\hline Volume of operation & $0.12\left[\mathrm{~m}^{3}\right]$ \\
\hline Rotation range & $\begin{array}{c}\text { Shoulder yaw: } \pm 150[\mathrm{deg}] \\
\text { Shoulder pitch: } \pm 90[\mathrm{deg}] \\
\text { Elbow pitch: } \pm 120[\mathrm{deg}]\end{array}$ \\
\hline Max. joint deflection & $\pm 30[\mathrm{deg}]$ \\
\hline
\end{tabular}

A rendered view of the compliant mechanism integrated in shoulder pitch and elbow pitch joints can be seen in Figure 2. A pair of compression springs transmits the motion from the servo shaft frame to the output link, or vice-versa, in both rotation directions. If the output frame is deflected clockwise (counter clockwise) then the right (left) spring is compressed while the opposite spring is free of load. A Murata SV01 potentiometer has been placed at the output frame, with its shaft attached to the servo shaft frame for deflection angle measurement. The output frame is supported by an $8 \mathrm{~mm} \varnothing$ shaft attached to servo shaft frame through an Igus EFOM-08 flange bearing in one side and by a $3 \mathrm{~mm} \varnothing$ screw in the back side of the servo. This compliant mechanism has not been considered in the shoulder yaw joint due to space limitations.

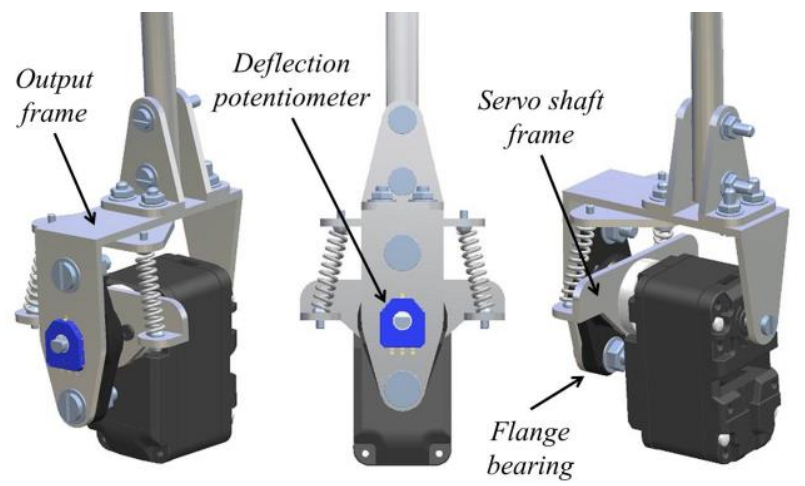

Figure 2. Rendered view of the compliant mechanism integrated in the shoulder pitch and elbow pitch joints.

Finally, note that although the arm has been represented upwards, it can be installed either upwards or downwards in the multi-rotor platform. This is a relevant issue, as the landing gear, the propellers and the floor impose motion constraints that must be considered for preventing collisions. 


\section{Compliant Finger Module}

The robot arm has been equipped with a 40 grams weight finger module [19], depicted in Figure 3, for object grasping and soft collision detection against walls or obstacles. The 3 DOF compliant and anthropomorphic finger is driven by a nylon tendon rolled up into a $6 \mathrm{~mm} \varnothing$ reel for finger flexion. Finger extension is achieved by means of an extension spring and the heat shrink tube that maintains the three finger bones tied together. The potentiometer attached to the metacarpophalangeal (MCP) joint allows the position control of this joint, while proximal inter phalange (PIP) and distal inter phalange (DIP) joints are under-actuated. As the MCP joint provides low stiffness, any frontal collision will cause a deviation in its position that can be easily detected, without affecting significantly the stability of the UAV. This can be exploited for navigation in narrow spaces, using the armfinger system for detecting obstacles in a similar way people do when they move through a room at night without seeing.

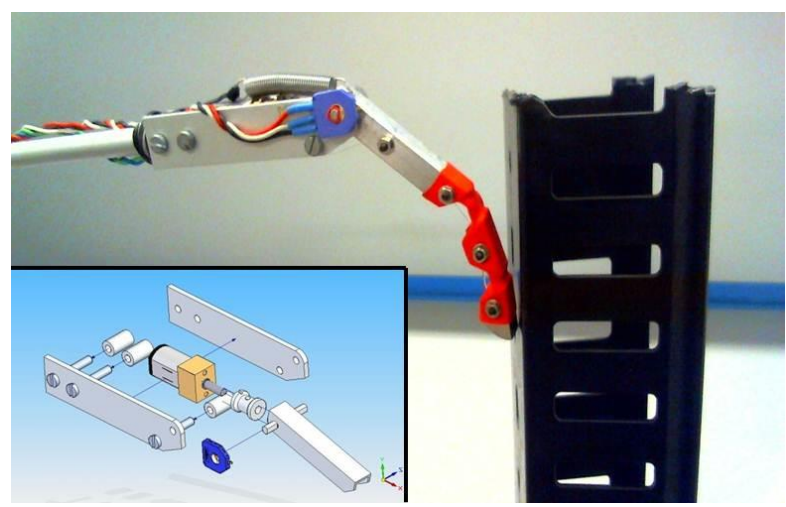

Figure 3. Compliant finger module deflected due to a frontal collision against an obstacle. MCP joint deflection is measured with a potentiometer.

\section{COMPLIANT FORCE-TORQUE ESTIMATION}

This section explains how to estimate the joint torque and the force at the wrist point from the deflection angle of the compliant mechanism incorporated to the shoulder pitch and elbow pitch joints. Torque estimation at shoulder yaw is not considered in this work as this joint is stiff. In the following, three variables related with joint position are defined:

- $\theta_{i}$ : servo shaft position of the $i$-th joint

- $q_{i}$ : output link position of the $i$-th joint

- $\Delta \theta_{i}$ : deflection angle of the $i$-th joint

where $i=\{1,2,3\}$ for shoulder yaw, shoulder pitch and elbow pitch joints, respectively. For clarity in the notation, it is imposed that $\Delta \theta_{1}=0$, so the first joint is treated in the same way the compliant joints.

\section{A. Torque Estimation based on Joint Deflection}

As mentioned before, the compliant torque estimation is based on the measurement of the deflection angle between the servo shaft and the output frame. The position of the output link can be expressed as the sum of the servo position and the joint deflection:

$$
q_{i}=\theta_{i}+\Delta \theta_{i}
$$

Consider now that diagram depicted in Figure 4 where the compliant joint is deflected clockwise so the right spring is compressed with a force proportional to its elongation:

$$
F_{\text {spring }}=K \cdot \Delta d=K \cdot[d(0)-d(\Delta \theta)]
$$

Here $K$ is the elastic constant and $d(\Delta \theta)=\left\|\boldsymbol{P}_{\mathbf{1}}-\boldsymbol{P}_{\mathbf{2}}\right\|$ is the distance between the contact points of the spring:

$$
\boldsymbol{P}_{\mathbf{1}}=\left[\begin{array}{l}
a \\
0
\end{array}\right] \quad \boldsymbol{P}_{\mathbf{2}}=\left[\begin{array}{l}
r \cdot \sin \left(\Delta \theta+\varphi_{0}\right) \\
r \cdot \cos \left(\Delta \theta+\varphi_{0}\right)
\end{array}\right]
$$

The torque generated by the spring is then:

$$
\tau_{\text {spring }}=F_{\text {spring }} \cdot r \cdot \sin \left(\Delta \theta+\varphi_{0}\right)
$$

The maximum deflection angle is determined by the minimum length of the spring when fully compressed. In the implemented mechanism, the value of the constants are $K_{2}=$ $2 \mathrm{~N} / \mathrm{mm}, K_{3}=1.7 \mathrm{~N} / \mathrm{mm}, a=20 \mathrm{~mm}, r=26 \mathrm{~mm}, \varphi_{0}=$ $35 \mathrm{deg}$

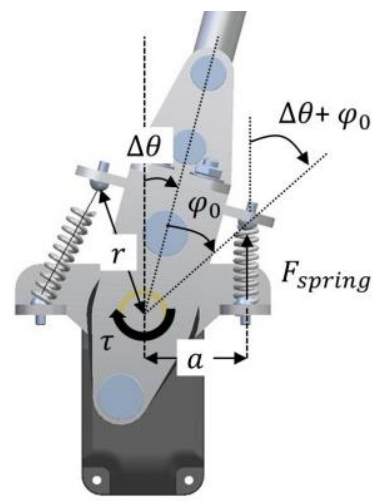

Figure 4. Variables involved in the torque estimation from joint deflection.

\section{B. Force-Torque Relationships}

As the compliant arm is expected to operate in contact with the environment, it is necessary to relate the contact forces at the end effector or wrist point w.r.t. joints torque, so the contact force can be controlled through joints deflection. Taking into account that only shoulder pitch and elbow pitch joints are compliant, then the problem is reduced to a 2-DOF manipulator, as shown in Figure 5. The torque in these two joints is related with the $\mathrm{XZ}$ components of the force at the wrist point in the following way:

$$
\left[\begin{array}{l}
\tau_{2} \\
\tau_{3}
\end{array}\right]=\left[\begin{array}{ll}
a & b \\
c & d
\end{array}\right] \cdot\left[\begin{array}{l}
F_{x} \\
F_{y}
\end{array}\right] ; \quad\left[\begin{array}{l}
F_{x} \\
F_{z}
\end{array}\right]=\frac{1}{\Delta}\left[\begin{array}{cc}
d & -b \\
-c & a
\end{array}\right] \cdot\left[\begin{array}{l}
\tau_{2} \\
\tau_{3}
\end{array}\right]
$$

where variables $a, b, c, d$ and $\Delta$ are defined as follows:

$$
\begin{gathered}
a=-L_{1} \cdot \cos \left(q_{2}\right)-L_{2} \cdot \cos \left(q_{2}+q_{3}\right) \\
b=L_{1} \cdot \sin \left(q_{2}\right)+L_{2} \cdot \sin \left(q_{2}+q_{3}\right) \\
c=-L_{2} \cdot \cos \left(q_{2}+q_{3}\right) \\
d=L_{2} \cdot \sin \left(q_{2}+q_{3}\right) \\
\Delta=-L_{1} \cdot L_{2} \cdot \sin \left(q_{3}\right)
\end{gathered}
$$

In the developed arm, the upper arm and forearm lengths are respectively $L_{1}=200 \mathrm{~mm}$ and $L_{2}=200 \mathrm{~mm}$. 


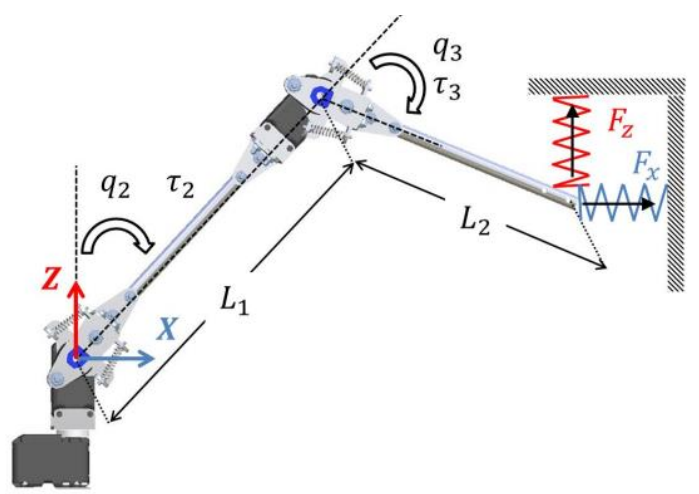

Figure 5. Geometric model considered for the force-torque relationships in static conditions with the wrist point in contact with a surface.

\section{MODELING AND CONTROL}

This section presents the equations of the kinematic and dynamic models for the developed arm. The design of the control scheme is preceded by a review of the tasks that the compliant arm is intended to perform. The dynamic modeling of the whole arm-UAV system is not considered in this work. The control of the compliant finger is detailed in [19].

\section{A. Kinematic Model}

Let $q_{1}, q_{2}$ and $q_{3}$ be the positions of the shoulder yaw, shoulder pitch and elbow pitch joints, respectively, and $L_{1}$ and $L_{2}$ the upper arm and forearm lengths. The position of the wrist point with respect to the reference frame attached to the base of the arm (see Figure 5) is given by:

$$
\left[\begin{array}{l}
x \\
y \\
z
\end{array}\right]=\left[\begin{array}{c}
r\left(q_{2}, q_{3}\right) \cdot \cos \left(q_{1}\right) \\
r\left(q_{2}, q_{3}\right) \cdot \sin \left(q_{1}\right) \\
L_{1} \cdot \cos \left(q_{2}\right)+L_{2} \cdot \cos \left(q_{2}+q_{3}\right)
\end{array}\right]
$$

where $r\left(\theta_{2}, \theta_{3}\right)$ is defined in the following way:

$$
r\left(q_{2}, q_{3}\right)=L_{1} \cdot \sin \left(q_{2}\right)+L_{2} \cdot \sin \left(q_{2}+q_{3}\right)
$$

The proposed configuration has analytical solution for the inverse kinematics, so the joint variables can be obtained from the desired Cartesian position:

$$
\left[\begin{array}{l}
q_{1} \\
q_{2} \\
q_{3}
\end{array}\right]=\left[\begin{array}{c}
\operatorname{atan} 2(y, x) \\
\cos ^{-1}\left(\frac{x^{2}+y^{2}+z^{2}+L_{1}^{2}-L_{2}^{2}}{2 \cdot L_{1} \cdot \sqrt{x^{2}+y^{2}}}\right) \\
\cos ^{-1}\left(\frac{x^{2}+y^{2}+z^{2}-L_{1}^{2}-L_{2}^{2}}{2 \cdot L_{1} \cdot L_{2}}\right)
\end{array}\right]
$$

Known the deflection of each joint, the position of the corresponding servo is obtained from Equation (1).

\section{B. Dynamic Model}

The equations of the dynamic model for a robotic arm with compliant joints can be decomposed in two parts [21]. On the one hand, the dynamics of the output links can be expressed in the usual form:

$$
\begin{gathered}
\boldsymbol{M}(\boldsymbol{q}) \ddot{\boldsymbol{q}}+\boldsymbol{C}(\boldsymbol{q}, \dot{\boldsymbol{q}}) \dot{\boldsymbol{q}}+\boldsymbol{G}(\boldsymbol{q})=\boldsymbol{\tau}+\boldsymbol{\tau}_{\text {ext }} \\
\boldsymbol{\tau}=\boldsymbol{K}(\boldsymbol{\theta}-\boldsymbol{q})+\boldsymbol{D}(\dot{\boldsymbol{\theta}}-\dot{\boldsymbol{q}})
\end{gathered}
$$

where $\boldsymbol{M}, \boldsymbol{C}$ and $\boldsymbol{G}$ represent the link inertia, centrifugal and Coriolis term, and the gravity component, respectively, $\boldsymbol{\tau}$ is the torque introduced by the compliant joint with $\boldsymbol{K}=$ $\operatorname{diag}\left(k_{i}\right)$ and $\boldsymbol{D}=\operatorname{diag}\left(d_{i}\right)$ being the elastic and friction constants for each joint, while $\boldsymbol{\tau}_{\text {ext }}$ is a torque generated from the action of external forces. This model assumes that the compliant joint behaves as a spring-damper system. On the other hand, the dynamics for the servos are described by:

$$
B(\boldsymbol{\theta}) \ddot{\theta}+\tau=\tau_{m}-\tau_{f}
$$

Here $\boldsymbol{B}$ is the inertia of servos shaft and frame, $\boldsymbol{\tau}_{\boldsymbol{m}}$ is the torque generated by the motor and $\boldsymbol{\tau}_{\boldsymbol{f}}$ is a friction term. As it can be seen, the common term in Equation (10) and Equation (12) is the torque supported by the compliant element, which transmit the torque generated by the motor to the output link.

As this work is focused in the estimation and control of the force-torque when there is a physical interaction between the aerial manipulator and the environment, for example when it is exerting a force against a wall or it is grasped, the inertial, Coriolis and centrifugal terms in this model have not been considered. However, it is necessary to remark that the position control of the arm may become unstable if the arm is moving freely and joint deflection is introduced in the control loop without taking into account theses terms. What is more, it would be necessary to model the perturbation that the motion of the UAV itself introduces over the compliant joint.

\section{Task Identification}

The design of the control scheme for the compliant arm is influenced by the tasks it is intended to perform. In general, the aerial manipulator may operate in two conditions: free motion (contactless), and interacting with the environment. For the first case it is desirable to provide high accuracy in the positioning of the end effector, and at the same time, the possibility of detecting sudden collisions or impacts, so the arm will have to move slowly to prevent joint deflection due to inertia. For the later, two types of interactions with the environment are considered: grasping an object attached to a base, such like a bar, and the application of pushing forces with the end effector in contact with a surface. The grasping on flight of a fixed object can be seen as a regulation problem in which the deflection angle of the compliant joints must be zero, acting over servos position for this purpose. For the control of the contact force between the end effector and the environment, the desired force is translated into a reference torque as described on Section III-B, measuring the current force in terms of the joint deflection.

Finally, the compliant finger module attached to the tip of the arm can be exploited, not only for grasping, but also for detecting and localizing obstacles on flight with negligible disturbance over the UAV. This is simply done monitoring the deflection of the MCP joint, which provides a very small stiffness, just defining a constant threshold for the detection.

\section{Control Scheme}

This works assumes that the wrist point or the tool center point of the arm is in contact with the environment and so inertial and centrifugal terms have less influence than gravity or external torques.

The proposed control scheme, illustrated in Figure 6, exploits the position controller embedded in all the Herkulex servos for the generation of joint trajectories with smooth 
variation of the velocity profile while controlling joint torque. The servo controller takes as input the desired goal position $\theta_{\text {ref }}$ along with the play time, that is, the desired time for reaching the reference position. The embedded controller generates a trapezoidal velocity profile, allowing smooth operation if the position references are sent to the servo at the midpoint of the playtime. The desired Cartesian position for the end effector $\mathbf{p}_{\text {ref }}$ is transformed into the joint position vector $\mathbf{q}_{\text {ref }}$ by applying Equation (9). This reference sent to the servos is obtained from Equation (1), adding a correction term for compensating the deflection of the compliant joints, $\theta_{c}$. A PID controller takes as input the difference between the torque reference $\boldsymbol{\tau}_{\text {ref }}$ and the external torque $\boldsymbol{\tau}_{\text {ext }}$ estimated from Equation (10). The adjustable threshold Dead Zone block acts as rejection filter, preventing that small deviations in the compliant joint affects the position controller. In the extreme case, it can be used as a switch for enabling or disabling the joint torque control mode. The inertial and centrifugal term in Equation (10) can be neglected if servo speed is slow enough so joint deflection is not significant.

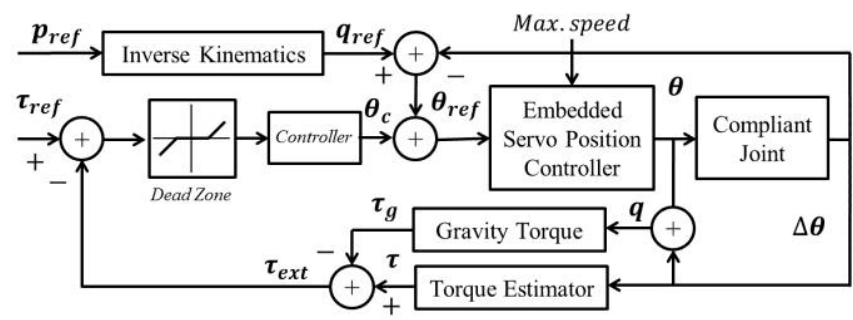

Figure 6. Joint position-torque control scheme in static (contact) conditions. The input references are end effector position reference, joints torque and maximum servo speed.

\section{EXPERIMENTAL RESULTS}

The developed compliant arm and compliant finger have been validated in a fixed base test bench. Three types of experiments have been performed: the characterization of the compliant joint, obstacle detection with the finger module and force control in contact with a vertical surface.

\section{A. Compliant Joint Characterization}

The joint torque-deflection static characteristic described in Section III-A has been evaluated numerically and through an identification experiment, obtaining that both variables are almost proportional in the operation range of the springs with a deflection angle under $30 \mathrm{deg}$. That is, the compliant joint behaves like a linear torsion spring. Two compression springs with constants $K_{1}=1.67 \mathrm{~N} \cdot \mathrm{mm}$ and $K_{2}=2 \mathrm{~N} \cdot \mathrm{mm}$ were tested in the shoulder pitch joint, exploiting the mass of the forearm and upper arm links $(0.2 \mathrm{Kg}$ with its center of mass at $185 \mathrm{~mm}$ from the shoulder pitch shaft) for generating the torque due to gravity. The equivalent torsion constants for the shoulder pitch and elbow pitch joints are then $K_{J 2}=28 \mathrm{~N}$. $\mathrm{mm} / \mathrm{deg}$ and $K_{J 3}=9 \mathrm{~N} \cdot \mathrm{mm} / \mathrm{deg}$

The dynamic response of the compliant mechanism is analyzed in terms of the step response. A $30 \mathrm{deg}$ amplitude step is applied over servo position, specifying different joint speeds, from $30 \mathrm{deg} / \mathrm{s}$ up to $50 \mathrm{deg} / \mathrm{s}$. As it can be seen in Figure 7, the oscillations in the deflection angle increase as the joint speed increases due to the inertial and centrifugal terms in Equation (10). On relevant conclusion derived from Figure 7 is that the maximum speed allowed for the servos must be under $38 \mathrm{deg} / \mathrm{s}$ so the compliant joints do not suffer for significant dynamic torques.
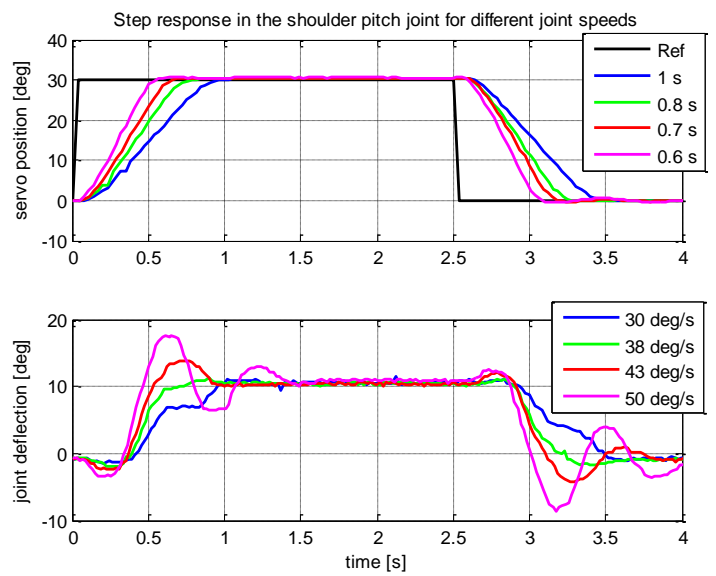

Figure 7. Step response in the shoulder pitch joint for different servo speeds. The influence of the inertia is more significant as servo speed increases.

\section{B. Soft Obstacle Localization with Compliant Finger}

In this experiment the finger module was incorporated in the forearm at $20 \mathrm{~cm}$ distance from elbow shaft to MCP joint. The arm, at fixed base, executed a $180 \mathrm{deg}$ scan around the shoulder yaw joint, increasing the radius from $20 \mathrm{~cm}$ up to 35 $\mathrm{cm}$ in the XY plane. The collision detection threshold for the compliant MCP joint was set to $15 \mathrm{deg}$. The position of the tool center point (the MCP joint) during the scan has been represented in Figure 8, where the black marks correspond to those points in which finger deflection exceeds the detection threshold. This functionality may be useful if the multirotor is navigating in a narrow space with obstacles, so potential collisions can be avoided using this sense of touch.

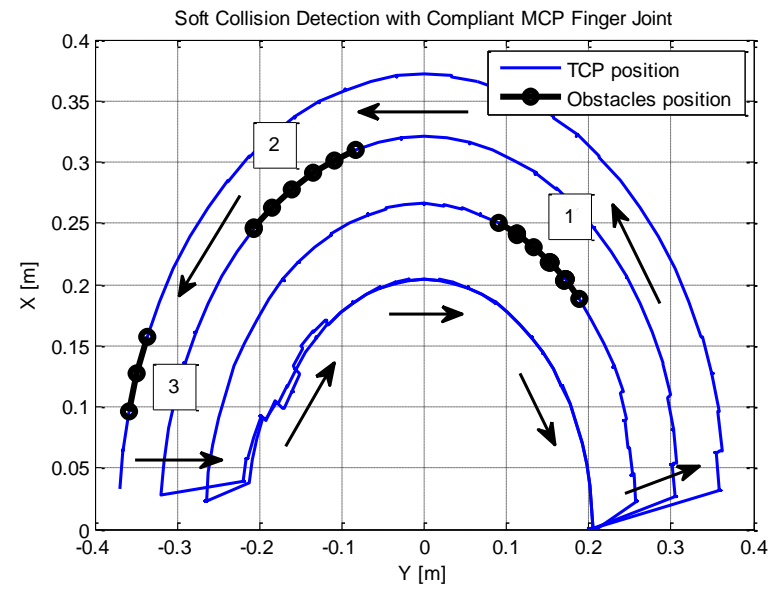

Figure 8. Tool Center Point (TCP) position with respect arm base (blue) and collision points (black) on the XY-plane. The position of the obstacle was moved from position 1 to position 3 during the experiment.

\section{Force Control in Fixed Base}

The control scheme described in Section IV-D is applied here for controlling the contact force at the wrist point. This capability is required for example in peg-in-hole tasks or in 
grasping situations. The configuration for the experiment is similar to the one shown in Figure 5, with a vertical contact surface at $18 \mathrm{~cm}$ distance from the base of the arm, which is initially extended $\left(q_{1}=0, q_{2}=0, q_{3}=0\right)$. The collision with the surface occurs when the elbow pitch servo moves from $\theta_{3}=0$ to $\theta_{3}=90 \mathrm{deg}$ and $q_{3} \approx 90 \mathrm{deg}$. The desired contact force in the $\mathrm{X}$-axis varies from $0.5 \mathrm{~N}$ to $1.5 \mathrm{~N}$, while the force in the Z-axis is set to zero. Figure 9 shows the reference and the estimated force in both axes along with the deflection angles in the elbow pitch and in the shoulder pitch joints. It must be noted that the contact force will be supported by the aerial platform when it is in flight.
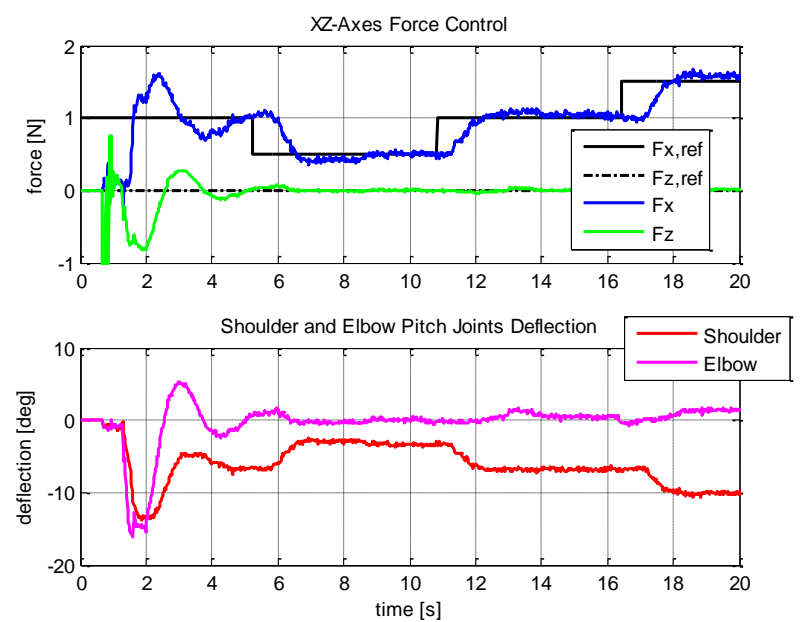

Figure 9. XZ-axes force reference and estimation (upper) and joints deflection (lower) with the wrist point in contact with a vertical surface. The collision occurs at $\mathrm{t}=0.7 \mathrm{~s}$ with $q_{2} \approx 0[\mathrm{deg}]$ and $q_{3} \approx 90[\mathrm{deg}]$.

\section{CONCLUSION}

This paper has presented the design and experimental validation of a 3 -DOF low weight $(0.3 \mathrm{Kg})$ robotic arm with compliance at shoulder pitch and elbow pitch joints intended for aerial manipulation applications involving contact forces with the environment. Joint torque and the contact force are estimated and controlled based on the measurement of joint deflection. A compliant anthropomorphic finger module is also considered for obstacle detection exploiting the low stiffness of the MCP joint. The performance of the armfinger system is demonstrated through experiments in fixed base test bench.

As future work, a full state estimator for the compliant arm will be developed and applied for improving the control in contact-free conditions. This involves estimating servo speed and acceleration for obtaining the inertia, centrifugal and Coriolis terms of the torque so they can be compensated.

\section{ACKNOWLEDGMENT}

This work has been founded by the AEROARMS Project founded by the European Commission under the H2020 ICT Programme (contract number 644271), and by the Spanish Ministerio de Educación, Cultura y Deporte FPU Program.

\section{REFERENCES}

[1] Kondak, K., Krieger, K., Albu-Schaeffer, A., Schwarzbach, M., Laiacker, M., Maza, I., .. \& Ollero, A. (2013). Closed-loop behavior of an autonomous helicopter equipped with a robotic arm for aerial manipulation tasks. Int. Journal of Advanced Robotic Systems, 10.

[2] Ruggiero, F., Trujillo, M. A., Cano, R., Ascorbe, H., Viguria, A., Perez, C., ... \& Siciliano, B. A multilayer control for multirotor UAVs equipped with a servo robot arm. In Robotics and Automation (ICRA), 2015 IEEE International Conference on (pp. 4014-4020).

[3] Jimenez-Cano, A. E., Martin, J., Heredia, G., Ollero, A., \& Cano, R. Control of an aerial robot with multi-link arm for assembly tasks. In Robotics and Automation, 2013 Int. Conf. on (pp. 4916-4921).

[4] Mellinger, D., Lindsey, Q., Shomin, M., \& Kumar, V. (2011, September). Design, modeling, estimation and control for aerial grasping and manipulation. In Intelligent Robots and Systems (IROS), 2011 IEEE/RSJ International Conference on (pp. 2668-2673).

[5] G. Garimella, M. Kobilarov. Towards model-predictive control for aerial pick-and-place. In Robotics and Automation (ICRA), 2015 IEEE International Conference on, May 2015, pp. 4692-4697.

[6] Keemink, A. Q., Fumagalli, M., Stramigioli, S., \& Carloni, R. (2012, May). Mechanical design of a manipulation system for unmanned aerial vehicles. In Robotics and Automation (ICRA), 2012 IEEE International Conference on (pp. 3147-3152).

[7] Caccavale, F., Giglio, G., Muscio, G., \& Pierri, F. (2014). Adaptive control for UAVs equipped with a robotic arm. Jeb, 3, I3.

[8] Antonelli, G., \& Cataldi, E. Adaptive control of arm-equipped quadrotors. Theory and simulations. In Control and Automation (MED), 2014 22nd Mediterranean Conference of (pp. 1446-1451).

[9] Ruggiero, F., Cacace, J., Sadeghian, H., \& Lippiello, V. (2014, May). Impedance control of VTOL UAVs with a momentum-based external generalized forces estimator. In Robotics and Automation (ICRA), 2014 IEEE International Conference on (pp. 2093-2099).

[10] Bellicoso, C. D., Buonocore, L. R., Lippiello, V., \& Siciliano, B. (2015, June). Design, modeling and control of a 5-DoF light-weight robot arm for aerial manipulation. In Control and Automation (MED), 2015 23th Mediterranean Conference on (pp. 853-858).

[11] Suarez, A., Heredia, G., \& Ollero, A. (2015, September). Lightweight compliant arm for aerial manipulation. In Intelligent Robots and Systems (IROS), 2015 International Conference on (pp. 1627-1632).

[12] Kim, S., Choi, S., \& Kim, H. J. Aerial manipulation using a quadrotor with a two dof robotic arm. In Intelligent Robots and Systems (IROS), 2013 IEEE/RSJ International Conference on (pp. 4990-4995).

[13] Korpela, C., Orsag, M., \& Oh, P. Towards valve turning using a dualarm aerial manipulator. In Intelligent Robots and Systems (IROS 2014), 2014 International Conference on (pp. 3411-3416).

[14] Eiberger, O., Haddadin, S., Weis, M., Albu-Schaffer, A., \& Hirzinger, G. (2010, May). On joint design with intrinsic variable compliance: derivation of the DLR QA-Joint. In ICRA (pp. 1687-1694).

[15] Yoon, S. S., Kang, S., Yun, S. K., Kim, S. J., Kim, Y. H., \& Kim, M. (2005). Safe arm design with MR-based passive compliant joints and viscoelastic covering for service robot applications. Journal of mechanical science and technology, 19(10), 1835-1845.

[16] Yuksel, B., Mahboubi, S., Secchi, C., Bulthoff, H. H., \& Franchi, A. Design, identification and experimental testing of a light-weight flexible-joint arm for aerial physical interaction. In Robotics and Automation (ICRA), 2015 International Conference on (pp. 870-876).

[17] Wisanuvej, P., Liu, J., Chen, C. M., \& Yang, G. Z. Blind collision detection and obstacle characterisation using a compliant robotic arm. In Robotics and Automation 2014 Int. Conf. on (pp. 2249-2254).

[18] Pounds, P. E., Dollar, A. M. (2014). Aerial grasping from a helicopter UAV platform. In Experimental Robotics (pp. 269-283). Springer.

[19] Suarez, A., Heredia, G., \& Ollero, A. (2016). Compliant and Lightweight Anthropomorphic Finger Module for Aerial Manipulation and Grasping. In Robot 2015: Second Iberian Robotics Conference (pp. 543-555). Springer International Publishing.

[20] Zollo, L., Siciliano, B., De Luca, A., Guglielmelli, E., \& Dario, P. (2005). Compliance control for an anthropomorphic robot with elastic joints: Theory and experiments. Journal of dynamic systems, measurement, and control, 127(3), 321-328.

[21] Albu-Schäffer, A., Ott, C., \& Hirzinger, G. (2007). A unified passivity-based control framework for position, torque and impedance control of flexible joint robots. The International Journal of Robotics Research, 26(1), 23-39. 